Georgia State University

ScholarWorks @ Georgia State University

\title{
Randomized Clinical Trial of Distraction for Infant Immunization
} Pain

Lindsey L. Cohen

Georgia State University, Ilcohen@gsu.edu

Jill E. MacLaren

Dalhousie University Faculty of Medicine, jill.chorney@dal.ca

Beverly L. Fortson

Centers for Disease Control and Prevention, hvj7@cdc.gov

Abby Friedman

Melissa DeMore

See next page for additional authors

Follow this and additional works at: https://scholarworks.gsu.edu/psych_facpub

Part of the Psychology Commons

\section{Recommended Citation}

Cohen, L. L., MacLaren, J. E., Fortson, B. L., Friedman, A., DeMore, M., Lim, C. S., Shelton, E., \& Gangaram, B. (2006). Randomized clinical trial of distraction for infant immunization pain. Pain, 125(1-2), 165-171. DOI: 10.1016/j.pain.2006.05.016

This Article is brought to you for free and open access by the Department of Psychology at ScholarWorks @ Georgia State University. It has been accepted for inclusion in Psychology Faculty Publications by an authorized administrator of ScholarWorks @ Georgia State University. For more information, please contact scholarworks@gsu.edu. 
Authors

Lindsey L. Cohen, Jill E. MacLaren, Beverly L. Fortson, Abby Friedman, Melissa DeMore, Crystal S. Lim, Elisabeth Shelton, and Balram Gangaram

This article is available at ScholarWorks @ Georgia State University: https://scholarworks.gsu.edu/psych_facpub/14 
Running head: DISTRACTION FOR INFANT PAIN

Randomized Clinical Trial of Distraction for Infant Immunization Pain

\author{
Lindsey L. Cohen ${ }^{\mathrm{a}}$ \\ Jill E. MacLaren ${ }^{\mathrm{b}}$ \\ Beverly L. Fortson ${ }^{c}$ \\ Abby Friedman ${ }^{\mathrm{d}}$ \\ Melissa DeMore \\ Crystal S. Lim ${ }^{\text {a }}$ \\ Elisabeth Shelton ${ }^{\mathrm{f}}$ \\ Balram Gangaram ${ }^{\mathrm{g}}$
}

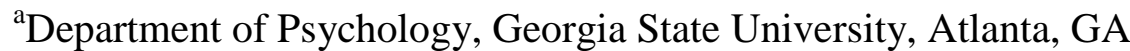

${ }^{\mathrm{b}}$ Department of Psychiatry and Human Behavior, Brown University, Providence, RI

${ }^{\mathrm{c}}$ Department of Psychiatry and Behavioral Sciences, Duke University, Durham, NC

${ }^{\mathrm{d}}$ Department of Psychology, West Virginia University, Morgantown, WV

${ }^{\mathrm{e}}$ Kennedy Krieger Institute, Johns Hopkins School of Medicine, Baltimore, MD

${ }^{\mathrm{f}}$ School of Nursing, West Virginia University, Morgantown, WV

${ }^{\mathrm{g}}$ Department of Pediatrics, Kapiolani Medical Center for Women and Children, University of Hawaii, Honolulu, HI

Number of text pages: 12

Number of figures: 1

Number of tables: 2

Corresponding Authors: Dr. Lindsey Cohen, Department of Psychology, Georgia State

University, Atlanta, GA 30302-5010

Phone: 404-651-1605

Fax: 404-651-1391

Email: 1lcohen@gsu.edu

Webpage: http://www2.gsu.edu/ wwwpsy/faculty/cohen.htm 


\begin{abstract}
Distraction has been shown to be an effective technique for managing pain in children; however, few investigations have examined the utility of this technique with infants. The goal of the current study was to investigate the effectiveness of movie distraction in reducing infants' immunization distress. Participants were 136 infants (range $=1$ to 21 months; $M=7.6$ months, $S D=5.0$ months) and their parents, all of whom were recruited when presenting for routine vaccinations. The parent-child dyads were randomly assigned to either a Distraction or Typical Care control condition. Infant and adult behaviors were assessed using a visual analog scale and a behavioral observation rating scale. Results indicated parents in the Distraction group engaged in higher rates of distraction than those in the Typical Care group, whereas there was no difference in the behavior of nurses in the Distraction and Typical Care groups. In addition, infants in the Distraction group displayed fewer distress behaviors than infants in the Typical Care group both prior to and during recovery from the injection. Findings suggest that a simple and practical distraction intervention can provide some distress relief to infants during routine injections.
\end{abstract}

Keywords: Acute pain; Distraction; Infants; Behavioral approaches; Immunizations 


\section{Randomized Clinical Trial of Distraction for Infant Immunization Pain}

A surge of research has revealed that infants experience pain similarly, if not more intensely, than adults (Porter et al., 1999). If untreated, pain can result in negative, long-term repercussions (Taddio, 1999; Young, 2005). The most common painful events during infancy are immunizations, which typically are conducted without pain management (Porter et al., 1997; Felt et al., 2000).

Nonpharmacologic pediatric pain management (i.e., cognitive-behavioral intervention) has few to no side-effects, is inexpensive and easy to use, and has been deemed "empirically supported" (Powers, 1999). These interventions typically include breathing retraining, relaxation, imagery, coping skills training, rehearsal, and reinforcement (Blount et al., 2003). They are not appropriate for infants as they require a degree of cognitive capacity not yet developed in infants. A promising intervention that does not require advanced cognitive skills is distraction.

Distraction is a potent anxiety and pain management tool for children's pain (for reviews, see Kleiber and Harper, 1999; Piira et al., 2002; DeMore and Cohen, 2005) but has received little evaluation in infants.

Two treatment studies have examined distraction pain management for infants. Cohen (2002) evaluated movie distraction for 2-month-olds to 3-year-olds receiving immunizations. Distraction was found to lower children's distress during the post-injection recovery phase but not during the anticipatory or procedural phases. In addition, results were demonstrated only on observational measures — treatment effects were not found on parent- or nurse-reports. More recently, Cramer-Berness and Friedman (2005) compared parent-directed toy distraction to a supportive care condition and a control group for infants age 2 months to 2 years. Parents in the supportive care condition used techniques that they had found effective in the past for reducing 
infant distress. Parents in the distraction condition were trained to use either games or toys as distraction during the injection. Results indicated that infants in the supportive care condition recovered more quickly from the immunization than infants in the control group, but there were no differences in infant distress in the distraction and control groups. These limited and inconsistent findings on distraction with infants suggest that continued examination of this intervention is warranted.

Any evaluation of interventions for procedural distress should consider changes in distress over the course of the procedure (Cohen, 2002). For example, anticipatory and recovery distress might be as intense or of longer duration than the distress experienced during the brief moment of the noxious stimulus (e.g., needle injection). Related, the efficacy of an intervention might vary by procedural phase (Blount et al., 2003).

In addition to demonstrating efficacy in research, the ultimate success of an intervention depends on the degree to which the intervention can be self-sustaining. Specifically, interventions should be cost-effective, time-efficient, and acceptable to the patients, parents, and staff. Unfortunately, few pain interventions meet these criteria. The purpose of this study was to examine the effectiveness of an easy-to-use and practical distraction intervention for reducing infants' immunization distress. Based on prior work (Cohen, 2002), we expected that distraction would exert its greatest effects during the anticipatory and recovery phases of the procedure.

\section{Method}

\section{Participants and Study Site}

This study was designed in accord with, and adheres to the guidelines detailed in, the Consolidated Standards of Reporting Trials statement (CONSORT; Altman et al., 2001; Moher et al., 2001; Stinson et al., 2003). Appropriate institutional approval was obtained prior to study 
initiation

Two health care facilities located in the southeastern United States participated. One facility was a university-affiliated medical center and the other was a private practice office employing three pediatricians. The two sites were approximately 20 miles apart. Data was collected at the medical center between October and November of 2002 and at the private practice between September and November of 2003. Inclusion criteria included any Englishspeaking families with an infant between 1 to 24 months presenting for routine checkups and vaccinations. Seven nurses performed the injections (four from the university-affiliated medical center and three from the private practice). The educational training of the nurses was roughly equivalent in the two locations: they were either registered or licensed practical nurses.

Power analyses using a medium to large effect size of .74, which was obtained in past research evaluating distraction for infant pain (Cohen, 2002), indicated that 48 participants would yield a power of .81 with a $t$-test (Faul and Erdfelder, 1992). We decided on a larger sample for two reasons. First, the Cohen (2002) finding is the only one suggesting that distraction works for infants. For example, Cramer-Berness and Friedman (2005) did not find distraction effective for infants. A larger sample would allow more confidence that non-results were not due to a lack of power. Second, our participants were going to be younger than those previously studied. Thus, we are not sure whether the large effect size found by Cohen (2002) is sufficiently applicable for this study population. Thus, we aimed for a sample of 120 , in which 60 patients would be randomized to each arm, which would yield power of .0 .85 to detect medium effect sizes.

A total of 161 families were approached to participate in the study; only $23(14.3 \%)$ declined. The families who declined to participate did so because of time constraints or they did 
not want to be videotaped. The final sample consisted of 136 infants ( 80 girls and 55 boys) who were primarily (90.4\%) Caucasian (Native American, 2.2\%; African American, 1.5\%; Hispanic, 0.7\%; and "other," 5.2\%) and ranged in age from 1 to 21 months $(M=7.6$ months, $S D=5.0$ months). The parents were 120 mothers and 16 fathers whose average age was 27 years $(M=$ 27.6, $S D=6.3)$. Most parents $(95.6 \%)$ described themselves as Caucasian $(3 \%$ were Native American; $1.5 \%$ African American) and middle class (annual income ranged from $\$ 0$ to $\$ 170,000, M=\$ 38,740, S D=\$ 29,158)$. Parents' educational level ranged from $8^{\text {th }}$ grade to postbaccalaureate, but most had completed one year of college $(M=13.1, S D=2.1)$. The final sample size of 136 participants yielded a power of 0.90 to detect a medium effect size.

\section{Measures}

Background information. A questionnaire to obtain demographic information about the parent (i.e., relation to child, age, gender, race, ethnicity, level of education, total family income, marital status) and the child (i.e., age, gender, race, and ethnicity) was completed by all families. This questionnaire also assessed medical information, including whether the child was currently on any medications, whether the child was his/her usual self upon presentation to the clinic, whether the child had received prior injections other than the regularly scheduled ones, and how distressed the child had been during previous immunizations. These latter data were collected to better understand and explain outliers in results, if needed.

Infant distress. Parents and nurses completed pre- and post-injection questionnaires.

Responses to questions were made utilizing a visual analog scale (VAS) consisting of $100 \mathrm{~mm}$ horizontal lines, anchored at the extremes with not distressed and very distressed. VAS scales commonly are used in pain studies, particularly because they have good reliability and validity and produce less bunching of scores than categorical scaling methods (Varni et al., 1990; 
McGrath, 1990). The pre-injection questionnaires asked parents and nurses to rate the current level of distress of the child, whereas the post-injection questionnaires asked parents and nurses to rate the distress level of the child during the injections.

The Measure of Adult and Infant Soothing and Distress (MAISD; Cohen et al., 2005) is a valid and reliable behavioral observation rating scale that was developed to evaluate the behaviors of infants, their parents, and nurses during painful pediatric medical procedures. Behaviors of interest for the infants included engage in distraction and distress (i.e., crying, screaming, or flailing). Parents' and nurses' distraction behaviors were coded.

Four trained undergraduate research assistants, blind to study hypotheses, coded behaviors from the videotaped immunizations. All behaviors were coded in five-second intervals, spanning from three minutes prior to when the nurse began cleaning the skin for the injection, until two minutes after the needle was removed or the child left the room, whichever came first. The index used for analyses was the proportion of 5-second intervals exhibiting the coded behavior, which was calculated by dividing the number of 5-second intervals in which the behavior was displayed by the total number of 5-second intervals.

Four undergraduate coders were trained on the MAISD using videotapes from other datasets until $98 \%$ agreement was obtained. Weekly meetings were held with the coders to review videotapes and to ensure that interrater agreement could be sustained. Each research assistant coded approximately 35 participants $(S D=6.45)$. Interrater reliability was examined on approximately $15 \%$ of the total sample (22 procedures), all of which were selected at random across all four coders. Percent agreement was coded for the infant, parent, and nurse behavioral codes. Percent agreement was selected because it is the recommended approach for evaluation of agreement for low base rate behavior (e.g., Cicchetti \& Feinstein, 1990; Spitzer \& Fleiss, 1974; 
Spitznagel \& Helzer, 1985; Uebersax, 1988). The mean percent agreement coefficients and standard deviations for each of the coded behaviors follow: infant engage in distraction, $M=$ 95.7, $S D=0.5$; infant distress, $M=93.0, S D=3.8$; parent distraction, $M=96.8, S D=1.3$; and nurse distraction, $M=97.2, S D=2.5$. Given that self-report is not available for infant pain, and parents' and nurses' ratings are subject to some biases, the MAISD scores were viewed as the primary measure of infant distress, with parents' and nurses' ratings as secondary indices.

\section{Procedure}

Data collection was conducted by trained graduate research assistants following specific and identical instructions at each site. Families were approached in the exam rooms after they had been seen by their pediatricians and before the nurses arrived with the immunizations. Informed consent was obtained at this time, background information was gathered, and families were informed as to the condition to which they had been assigned. Families were randomly assigned to study conditions (i.e., either Distraction or Typical Care) in accord with a random number table. The condition assignment was kept concealed in a binder and revealed after the patient agreed to participate. Videotaping began after families provided informed consent to be

in the study. Upon arrival into the exam room, the nursing staff was informed as to the condition in which the child had been placed. After the immunizations were complete, parents and nurses completed the post-injection questionnaires.

Typical care. In the Typical Care condition, the parent and nurse were encouraged to interact with the infants in their usual manner and use whatever techniques they commonly use to calm the infant. Although it was expected that some naturally occurring distraction may occur, no movie or toy distraction was provided to the infants. Parents and nurses also were expected to engage in comforting behaviors, reassurance, information provision, empathy, and apologizing to 
the child, which are behaviors commonly exhibited by adults during children's painful medical procedures (e.g., Blount et al., 1990; Manne et al., 1992; Cohen et al., 1997).

Distraction. Prior to data collection, nurses engaged in a brief intervention-training program in which the primary investigator or the research assistants described and modeled the distraction techniques (see Cohen et al., 1997). Prior to the injections, parents also briefly were instructed in distraction techniques that may be helpful for the child. During the immunization, a DVD movie, either Sesame Street ${ }^{\circledR}$ or Teletubbies ${ }^{\circledR}$ (based on the choice of the parents), was played on a handheld portable DVD player positioned approximately 6 inches from the child. Parents were instructed that they could redirect the child's attention to the video using animated gestures or speech (e.g., "Big bird is singing you a song!" or "Look at that!”). The researchers periodically reminded the nurses to adhere to the condition parameters. Although the flexibility in the intervention resulted in different distraction for different participants, the treatment was designed to be as naturalistic as possible to increase external validity and to ensure that little additional time was necessary when using the intervention. Due to the nature of the study protocol, the nurses performing the injections were not blind to study condition; however, they were not informed about the hypotheses of the investigation.

Results

\section{Data Analysis Overview}

Analyses were conducted in a series of steps. First, preliminary analyses were conducted to ensure that treatment groups and data collection sites were roughly similar on demographic variables, and to examine relations between age of participant and outcome. Second, treatment integrity analyses were conducted to determine whether the nurses and parents provided distraction as trained in the Distraction condition. Third, a 3 (Phase) x 2 (Condition) mixed 
repeated measures analysis of variance (ANOVA) was conducted to evaluate treatment effects across procedural phases.

Due to technical difficulties with video equipment ten (7.4\%) participants did not have video data and therefore could not be coded for infant distress, infant engage in distraction, nurse distraction, or parent distraction. These data were left as missing points in analyses and other compensatory actions were not taken (e.g., inserting a mean value).

Preliminary Analyses

Between treatment groups. Preliminary analyses were conducted to determine whether there were any significant between-group differences on demographic variables. Chi-square analyses (see Table 1) indicated no differences between groups on parent and infant race and infant gender. Parent gender was significantly different between groups with a higher proportion of male parents in the Distraction condition than in the Typical Care condition. $T$-tests revealed that there were no significant differences between conditions on child age, family income, parent education level, parent-report of infants' prior distress during immunizations, and parent-report of the amount of distress the infant experienced pre-procedure (Table 1).

Between sites. Preliminary analyses also were conducted to determine whether there were any significant between-site differences on demographic variables. Chi-square analyses (see Table 1) indicated no differences between groups on parent and infant race or parent and infant gender. $T$-tests revealed that there were no significant differences between sites on child age, parent education level, parent-report of infants' prior distress during immunizations, and parentreport of the amount of distress the infant experienced pre-procedure (Table 1). Results indicated a significant difference between sites on family income, however, with families at the private 
pediatrician's office having significantly higher incomes than those at the university-affiliated office.

Age relations. Correlational analyses indicated no significant relations between child age and any dependent variable (i.e., nurse and parent-rated distress, observational distress, engagement in distraction).

\section{Treatment Integrity}

In order to examine the degree to which parents and nurses adhered to training in each of the conditions and whether infants engaged in the distraction, treatment integrity analyses were conducted. Two $t$-tests were performed to compare nurse distraction and parent distraction across treatment groups. Results indicated that parents in the Distraction group performed significantly more distracting behavior than parents in the Typical Care group, $t(122)=2.80, p<$ .05; however, no significant differences were found between groups on nurse distracting behavior, $t(124)=1.15, n s$. Although these analyses indicate that groups did not differ on nurse behavior, the nature of the distraction stimulus and parent behavior seemed to be effective in engaging infants in distraction as infants in the Distraction condition were significantly more engaged in distraction than infants in the Typical Care condition, $t(123)=10.41, p<.05$.

\section{Treatment Effects}

Observational. A mixed repeated measures ANOVA was conducted to examine the effects of treatment group on infant distress over the course of the injection procedure (see Figure 1). Results of this ANOVA indicated a significant main effect of Phase, $F(2,216)=$ 44.04, $p<.05$, and a significant main effect of Treatment Group, $F(1,108)=9.91, p<.05$. The interaction between Phase and Treatment Group was non-significant, $F(1,108)=3.24$, $n s$. As expected from earlier group analyses, follow-up paired samples $t$-tests indicted that infants in the 
Distraction group evidenced less distress than infants in the Typical Care group during the anticipatory, $t(109)=2.02, p<.05$, and recovery phases, $t(123)=2.35, p<.05$. There was a nonsignificant difference in infant distress between the Distraction and Typical Care groups during the procedure phase, $t(124)=1.49, n s$. In terms of Phase analyses, follow-up paired samples $t$ tests indicated that infants displayed significantly more distress in the procedure phase than in the anticipatory phase, $t(110)=10.5, p<.05$. Distress in the recovery phase was not significantly different from distress in the procedure phase, $t(124)=.61, n s$, but was significantly higher than in the anticipatory phase, $t(109)=6.73, p<.05$.

Parent and nurse report. Descriptive statistics for infant distress by treatment group are shown in Table 2. $T$-tests were conducted to examine treatment effects on parent- and nursereported infant distress. No significant differences between Distraction and Typical Care groups were evident on either measure.

\section{Discussion}

Overall, results suggest that distraction is effective in reducing infants' behavioral distress, both in the anticipatory and recovery phases of the immunization. These findings are consistent with a prior evaluation of distraction for infant immunization distress (Cohen, 2002) and older children's procedural pain (for reviews, see Kleiber and Harper, 1999; Piira et al., 2002; DeMore and Cohen, 2005).

Although results suggest that distraction is helpful to infants, there are important details in this study that deserve attention. First, the treatment integrity analyses revealed that nurses did not perform significantly more distraction whether working with infants in the Distraction or Typical Care conditions. Despite the lack of differences in nurse behavior, distress-reducing effects of distraction were evident. Thus, we are left to assume that the beneficial effects of the 
treatment are explained by a) the distracting quality of the movies in and of themselves, and b) the coaching of the parents. The importance of the quality of the novel stimuli is consistent with a prior study finding that the distraction stimuli might be sufficient even in the absence of adult coaches (MacLaren and Cohen, 2005). Parent behavior also may account for the positive effects of the intervention. Despite the lack of differences in nurse distraction across conditions, differences in parent behavior across conditions were evident. Specifically, parents engaged in significantly more distracting behavior in the Distraction condition than in the Typical Care condition. This result is promising as parents received minimal training and had no in vivo encouragement to engage in distraction during the procedure. Further, the low rates of nurse distraction do not indicate that nurses modeled appropriate coaching behavior, as has been found in prior studies (e.g., Cohen et al., 1997). Thus, it is likely that the presence of the movie served as a cue for parent behavior. This is especially encouraging because it suggests that parents, who are salient figures in their infants' lives, are able to engage in helpful behavior. It should be noted that some research has indicated otherwise (e.g., Lewis and Ramsay). Analyses of infant distraction behaviors provide further support for the argument that the movie and parent coaching were sufficient to engage the infants' attention.

It is important to note that painful medical procedures have discrete phases with distinct dynamics operating in each (for a discussion, see Blount et al., 2003). As such, it is important to evaluate interventions on the basis of these phases. In the current project, procedures were divided into anticipatory, injection, and recovery phases, and infants' distress and the differential effectiveness of distraction in each of these phases was examined. Examined in this manner, it is clear that infants display relatively low distress prior to the injection, but that distraction is still effective in reducing distress further. Not surprisingly, infants' distress increases sharply during 
the injection and may not be as receptive to distraction during this phase. Infants' distress during recovery from the injection showed an interesting pattern. In the Typical Care condition, infants' distress actually increased beyond that evidenced during the injection. Conversely, distress decreased during recovery in infants in the Distraction condition. These results suggest that distraction is most effective prior to and for several minutes following the painful event.

Given the behavioral differences across groups, which were noted using the observational scale, it is troublesome that neither the nurses nor the parents rated infants in the Distraction group as less distressed than infants in the Typical Care group. Nurses' ratings may be explained by competing demands, as they may be focused on administering the injection, and therefore unable to carefully evaluate infant distress. The involvement in medical care also might provide an explanation for nurses' low rate of distraction coaching behavior. As for parents, it is likely that each parent evaluates his/her own child's distress in reference to a perceived baseline for that individual child. In line with this explanation, a number of studies have found poor agreement across parents', nurses', and children's ratings of pediatric pain (e.g., Chambers et al., 1998; Manne et al., 1992). Lastly, it might be that both the nurses and the parents are evaluating only the injection phase distress when completing the visual analog scale, which was not different across groups on the behavioral measure. In fact, parent report of infant distress demonstrates a small but significant correlation with injection phase behavioral distress, $r(128)=$ $.205, p=.021$, but not anticipatory or recovery phase distress. Nurse ratings did not correlate with any phase of behavioral distress. It is possible that more detailed questions, such as querying parents and nurses about distress in different phases of the injection, might better describe infants' distress reactions. Although there were no group differences, it is important to 
recognize that nurses and parents rated infants at the high end of the distress scale. Thus, additional pain management care and research is warranted.

Limitations of the current study should be noted. First, although it improved the internal validity of the study, external validity was compromised by the homogenous sample of predominantly Caucasian families from the Southeastern United States all undergoing routine immunizations. Future investigations of distraction for infant distress might enroll samples with diverse ethnic backgrounds undergoing a variety of distressing events. It is unfortunate that the coders could not be blinded to study conditions; both audio and visual aspects, that might have cued the coders to the conditions, were required for coding. Future studies might employ other types of distractions that are less visible on videotape to allow blind coding. Given that selfreport is not available for infants, physiological measures should be considered (e.g., cortisol, heart rate); however, each should be evaluated for validity, reliability, as well as practicality (for a review, see Sweet and McGrath, 1998). In addition, rate of pacifier sucking is a viable and unique measure that might be useful. Lastly, behavioral scales are critical for assessing distress in infants, and researchers should closely evaluate the literature as there are a number available (for a review, see McGrath, 1998).

In conclusion, distraction proved to decrease infants' behavioral distress during the anticipatory and recovery phases of immunization injections. Clearly additional work is needed to extend these findings in this neglected area of study, and additional interventions are needed to target infants' injection phase distress; however, these findings suggest that a cost-effective, time-efficient, and easy-to-use behavioral intervention can provide some comfort to infants during these routine distressing medical visits. 


\section{Acknowledgements}

This research was supported by a grant awarded by the Mayday Fund to Dr. Lindsey Cohen. 


\section{References}

Altman DG, Schulz KF, Moher D, Egger M, Davidoff F, Elbourne D, et al. The revised CONSORT statement for reporting randomized trials: Explanation and elaboration. Ann Intern Med 2001;13:663-94.

Blount RL, Piira T, Cohen LL. Management of pediatric pain and distress due to painful medical procedures. In: Roberts MC, editor. Handbook of pediatric psychology, $3^{\text {rd }} \mathrm{ed}$. Guilford Press;2003.

Blount RL, Sturges JW, Powers SW. Analysis of child and adult behavioral variations by phase of medical procedure. Behav Ther 1990;21:33-48.

Chambers, CT, Reid GJ, Craig KD, McGrath PJ, Finley GA. Agreement between child and parent report of pain. Clin J Pain 1998;14:336-342.

Cicchetti DV, Feinstein AR. High agreement but low kappa: II. Resolving the paradoxes. J Clin Epid 1990;43:551-558.

Cohen LL. Reducing infant immunization distress through distraction. Health Psychol 2002;21:207-11.

Cohen LL, Bernard RS, McClellan CB, MacLaren, JE. Assessing medical room behavior during infants' painful procedures: The measure of adult and infant soothing and distress (MAISD). Children's Health Care 2005;34:81-94.

Cohen LL, Blount RL, Panopoulos G. Nurse coaching and cartoon distraction: An effective and practical intervention to reduce child, parent, and nurse distress during immunization. J Pediatr Psychol 1997;22:355-70.

Cramer-Berness LJ, Friedman AG. Behavioral interventions for infant immunization. Childrens Health Care 2005;34:95-111. 
DeMore M, Cohen LL. Distraction for Pediatric Immunization. J Clin Psychol Med Settings 2005;12:281-92.

Faul F, Erdfelder E. GPOWER: A priori, post-hoc, and compromise power analyses for MS DOS-software program. Bonn University, Department of Psychology;1992.

Felt BT, Mollen E, Diaz S, Renaud E, Zeglis M, Wheatcroft G, et al. Behavioral interventions reduce infant distress at immunization. Arch Pediatr Adolesc Med 2000;154:719-24.

Kleiber C, Harper DC. Effects of distraction on children's pain and distress during medical procedures: A meta-analysis. Nursing Res 1999;48:44-9.

Lewis M, Ramsay DS. Effect of maternal soothing on infant stress response. Child Dev 1999;70:11-20.

MacLaren JE, Cohen LL. A comparison of distraction strategies for venipuncture distress in children. J Pediatr Psychol 2005;30:387-96.

Manne SL, Bakeman, R, Jacobsen PB, Gorfinkle K. Adult - child interaction during invasive medical procedures. Health Psychol 1992;11:241-9.

Manne SL., Jacobsen PB, Redd WH. Assessment of acute pediatric pain: Do child self-report, parent ratings, and nurse ratings measure the same phenomenon? Pain 1992:48:45-52.

McGrath PJ. Behavioral measures of pain. In: Finley GA, McGrath PJ, editors. Measurement of pain in infants and children, Progress in pain research and management, Vol. 10. IASP Press; 1998

Moher D, Schulz KR, Altman DG. The CONSORT statement: Revised recommendations for improving the quality of report of parallel groups randomized trials. BMC Med Res Methodol 2001;1:2. http://www.biomedcentral.com/1471-2288/1/2

Piira T, Hayes B, Goodenough B. Distraction methods in the management of children's pain: An 
approach based on evidence or intuition? The Suffering Child 2002;1:Article \#3. http://www.thesufferingchild.net/issues/issue01/03/index.htm

Porter FL, Wolf CM, Gold J, Lotsoff D, Miller JP. Pain and pain management in newborn infants: A survey of physicians and nurses. Pediatr 1997;100:626-32.

Porter FL, Grunau RE, Anand KJS. Long-term effects of pain in infants. J Dev and Behav Pediatr 1999;20:253-61.

Powers SW. Empirically supported treatments in pediatric psychology: Procedure-related pain. J Pediatr Psychol 1999;24:131-45.

Spitzer R, Fleiss J. A re-analysis of the reliability of psychiatric diagnosis. Brit J Psychiatr 1974:341-347.

Spitznagel EL, Helzer, JE. (1985). A proposed solution to the base rate problem in the kappa statistic. Arch Gen Psychiatr 1985;42:725-728.

Sweet SD, McGrath PJ. Physiological measures of pain. In: Finley GA, McGrath PJ, editors. Measurement of pain in infants and children, Progress in pain research and management, Vol. 10. IASP Press; 1998.

Taddio A. Effects of early pain experience: The human literature. In: McGrath PJ, Finley GA, editors. Chronic and recurrent pain in children and adolescents, Progress in pain research and management, Vol. 13. IASP Press; 1999.

Uebersax JS. Validity inferences from interobserver agreement. Psychol Bul 1988;104:405-416.

Stinson JN, McGrath PJ, Yamada JT. Clinical trials in the Journal of Pediatric Psychology: Applying the CONSORT statement. J Pediatr Psychol 2003;28:159-67.

Varni JW, Blount RL, Waldron SA, Smith AJ. Management of pain and distress. In: Roberts MC, editor. Handbook of pediatric psychology, $2^{\text {nd }}$ ed. Guilford Press; 1995. 
Young KD. Pediatric procedural pain. Ann Emerg Med 2005;45:160-71. 

Table 1. Demographic Variables by Condition

\begin{tabular}{|c|c|c|c|c|c|c|}
\hline & \multicolumn{2}{|c|}{ Condition } & \multirow[b]{2}{*}{$\begin{array}{l}\text { Comparison } \\
\text { Statistic }\end{array}$} & \multicolumn{2}{|c|}{ Site } & \multirow[b]{2}{*}{$\begin{array}{l}\text { Comparison } \\
\text { Statistic }\end{array}$} \\
\hline & Typical Care & Distraction & & University & Private & \\
\hline Child Race (\% Caucasian) & $88.1 \%$ & $92.6 \%$ & $X^{2}(4)=4.74$ & $91.0 \%$ & $89.7 \%$ & $X^{2}(1)=4.61$ \\
\hline Parent Race (\% Caucasian) & $95.6 \%$ & $95.6 \%$ & $X^{2}(2)=3.00$ & $95.6 \%$ & $95.6 \%$ & $X^{2}(2)=.000$ \\
\hline Child Age (months) & 7.7 & 7.5 & $t(130)=.203$ & 7.9 & 7.3 & $t(130)=.675$ \\
\hline Annual Family Income & 35439.1 & 42170.6 & $t(102)=1.18$ & 28971.2 & 48892.2 & $t(102)=3.69 *$ \\
\hline Infant Pre-procedure Distress ${ }^{\text {a }}$ & 12.3 & 13.8 & $t(134)=.367$ & 11.8 & 14.3 & $t(134)=.663$ \\
\hline
\end{tabular}

${ }^{\mathrm{a}}$ VAS scores, range 0 (no distress) to 100 (worst distress).

$* p<.01$. 
Table 2. Infant distress by condition

\begin{tabular}{|c|c|c|c|c|c|c|}
\hline & \multicolumn{2}{|c|}{ Typical Care } & \multicolumn{2}{|c|}{ Distraction } & \multirow[b]{2}{*}{ Effect Size } & \multirow[b]{2}{*}{$t(\mathrm{df})$} \\
\hline & $M$ & $S D$ & $M$ & $S D$ & & \\
\hline MAISD Parent Distraction & .033 & .065 & .167 & .346 & .652 & $2.80(122)^{*}$ \\
\hline MAISD Nurse Distraction & .030 & .067 & .044 & .069 & .206 & $1.15(124)$ \\
\hline MAISD Infant Engage in Distraction & .029 & .063 & .371 & .251 & 2.178 & $10.41(123)^{*}$ \\
\hline MAISD Anticipatory Distress & 0.17 & 0.31 & 0.06 & 0.21 & .423 & $2.02(109) *$ \\
\hline MAISD Injection Distress & 0.57 & 0.40 & 0.38 & 0.46 & .442 & $1.49(124)$ \\
\hline MAISD Recovery Distress & 0.60 & 0.61 & 0.31 & 0.38 & .586 & $2.35(123)^{*}$ \\
\hline MAISD Total Procedure Distress & 0.46 & 0.38 & 0.32 & 0.33 & .394 & $1.81(124) *$ \\
\hline Parent-report of Infant Distress & 70.5 & 27.9 & 70.9 & 26.0 & .015 & $0.10(134)$ \\
\hline Nurse-report of Infant Distress & 67.0 & 30.4 & 61.8 & 31.9 & .167 & $0.97(134)$ \\
\hline
\end{tabular}




\section{Randomized Clinical Trial 24}

Figure Caption

Figure 1. Observed infant distress by Phase and Treatment Group 


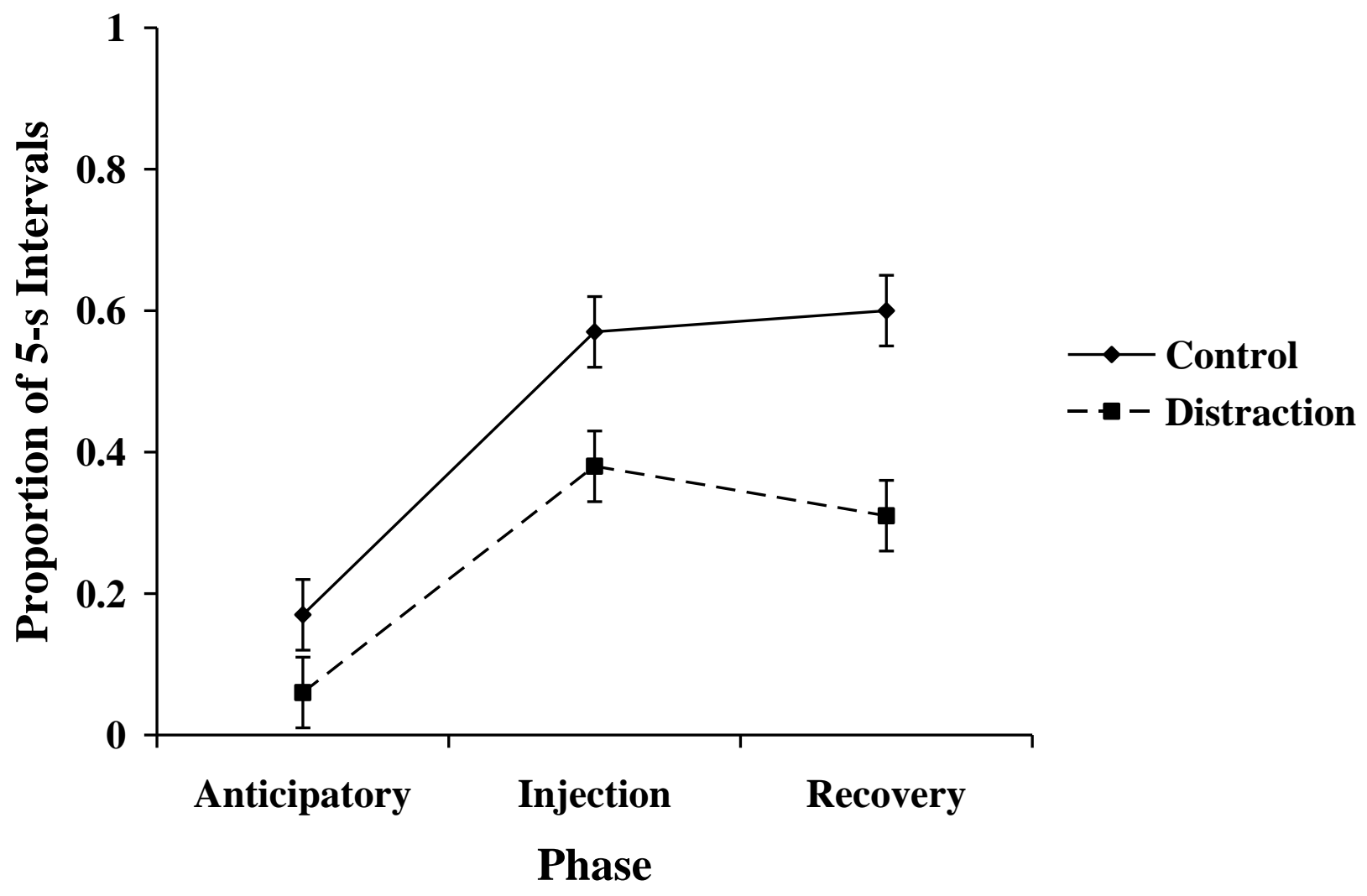

